\title{
A SOCIOLOGIA DA INFÂNCIA E OS DESENHOS INFANTIS - UMA CONTRIBUIÇÃO SOCIOLÓGICA À EDUCAÇÃO ${ }^{1}$
}

\section{THE SOCIOLOGY OF CHILDHOOD AND CHILDREN'S DRAWINGS - A SOCIOLOGICAL CONTRIBUTION TO EDUCATION}

\author{
BORDIN, Francine Borges \\ francine.bb1988@hotmail.com \\ UFPel - Universidade Federal de Pelotas \\ BUSSOLETTI, Denise \\ denisebussoletti@gmail.com \\ UFPel - Universidade Federal de Pelotas
}

\begin{abstract}
RESUMO Este artigo busca revisar alguns conceitos oriundos da sociologia da infância, a fim de contribuir para a construção de um campo disciplinar, que investigue os desenhos infantis a partir de uma perspectiva sociológica. Dialogamos com os sociólogos Manuel Sarmento através do conceito de cultura da infância e William Corsaro com o conceito de reprodução interpretativa. Consideramos os desenhos infantis como importante fonte de acesso às culturas da infância e como uma forma de crítica à cultura contemporânea, permitindo-nos ampliar nosso conhecimento sobre as peculiaridades das crianças e suas infâncias, bem como aperfeiçoar nosso trabalho de pesquisa e de docência.
\end{abstract}

PALAVRAS-CHAVE: Culturas Infantis. Desenhos Infantis. Educação Infantil. Sociologia da Infância.

ABSTRACT This article seeks to review some concepts from the sociology of childhood in order to contribute to the construction of a field subject, to investigate the children's drawings from a sociological perspective. We dialogue with sociologists Manuel Sarmento through the concept of culture of childhood and William Corsaro with the concept of interpretive reproduction. We consider the children's drawings as an important source of access to the cultures of childhood and as a form of critique of the contemporary culture, allowing us to expand our knowledge about the peculiarities of children and their childhoods and improve our research and teaching. KEYWORDS: Children's Drawings. Culture of Childhood. Early Childhood Education. Sociology of Childhood.

\footnotetext{
${ }^{1}$ Este artigo é resultado do diálogo entre trabalho de conclusão de curso de Especialização em Educação Infantil (2013) e dissertação de Mestrado em Educação (2014), realizados na Universidade Federal de Pelotas.
} 


\title{
1 INTRODUÇÃO
}

A sociologia da infância tem se constituído como um campo de estudo específico sobre a infância, contribuindo para a construção de novas referências nos novos estudos sociais da criança (REVISTA EDUCAÇÃO E SOCIEDADE, 2005). Não abordaremos aqui a constituição social e histórica da infância, mas apresentaremos a revisão de dois conceitos oriundos da sociologia da Infância culturas da infância, de Manuel Sarmento $(2002,2003)$ e reprodução interpretativa, de William Corsaro (2011) - buscando compreender de que forma esses conceitos podem colaborar para interpretar os desenhos infantis ou problematizar essa interpretação. Nessa perspectiva, examinamos a dissertação de Mestrado em Sociologia da Infância, "Desenho Infantil - Modos de interpretação do mundo e simbolização do real, um estudo em Sociologia da Infância”, de Zélia F. F. Gomes (2009).

\begin{abstract}
Em síntese, as diferentes correntes, abordagens e teorias presentes na Sociologia da Infância confluem num conjunto de aspectos que constituem marcadores do campo teórico. Linhas que assinalam a especificidade de uma reflexão teórica sobre a infância, ainda que algumas dessas linhas não sejam exclusivas, isto é, sejam de algum modo comum a outros campos disciplinares (especialmente a Antropologia da Infância, a Psicologia ou a Sociologia da Educação) e sejam diferenciadamente enfatizadas pelas diversas abordagens. É o conjunto desses pontos que estabelece as bases conceptuais da disciplina, e eles constituem a súmula dos contributos teóricos produzidos. Apesar de confluente, a partir deles estabelecem-se linhas de fuga, que assinalam a heterogeneidade do campo e definem as rupturas teóricas entre paradigmas, teorias e abordagens. (SARMENTO; GOUVEA, 2008, p.32).
\end{abstract}

Nosso objetivo neste texto é realizar uma reflexão teórica e sociológica sobre os desenhos infantis a partir de dois trabalhos de pesquisa: o primeiro diz respeito ao trabalho de conclusão do curso de Especialização em Educação Infantil (BORDIN, 2013) em que se realizou um levantamento teórico relacionando os desenhos infantis e a sociologia da infância e o outro estudo, realizado para o Mestrado em Educação (BORDIN, 2014), realizou um trabalho de campo com crianças de cinco a seis anos em uma escola pública de educação infantil. Não 
abordaremos aqui os desenhos e suas peculiaridades, mas levantaremos as possibilidades trazidas por eles.

Vale ressaltar que a infância está, há mais de um século, no centro das reflexões de importantes áreas do conhecimento, como é o caso da Psicologia. Nesta longa jornada muitas contribuições poderiam ser destacadas, como também muitas críticas. No entanto, é importante salientar que nosso objetivo neste texto não é o de expor ou contrapor as contribuições da Psicologia para a Sociologia da Infância, em que pese este reconhecimento. Trata-se de destacar o compromisso atual e renovado da Sociologia da Infância em evidenciar o lugar da infância nas Ciências Sociais, da mesma forma que tem sido feito com outras categorias sociológicas. Compromisso que embora se estruture pela premissa da interdisciplinaridade, para fins de nossos objetivos restringe seu enfoque apenas à Sociologia da Infância.

Assim sendo, nosso artigo se estruturará em quatro partes principais: na primeira trataremos do conceito de culturas da infância; na segunda abordaremos o conceito de reprodução interpretativa; na terceira buscaremos expor sobre os desenhos infantis numa perspectiva socioantropológica, tal qual exposta por Sarmento (2011); e, na quarta, levantaremos algumas contribuições à pesquisa e à prática educativa. Em seguida, nas conclusões finais, relacionaremos a importância destes estudos para a educação na contemporaneidade.

Faz-se necessário inicialmente esclarecer, que a infância neste texto é considerada como uma categoria social de tipo geracional (SARMENTO, 2003), sendo esta uma forma estrutural da sociedade (CORSARO, 2011). Como parte da estrutura da sociedade, torna-se necessário conhecer suas peculiaridades e para isso, julgamos necessário conhecer a relação entre os conceitos acima citados e os desenhos infantis (e sua produção). As teorizações que nos permitem pensar sobre os desenhos produzidos pelas crianças nos levam a considerar que estes podem se configurar como uma forma de crítica sobre a cultura e como um contraponto que nos permite pensar a infância contemporânea, buscando compreender acerca das produções infantis e do espaço das crianças em nossas pesquisas e práticas educativas. 


\section{CULTURAS DA INFÂNCIA}

As culturas da infância são caracterizadas por Sarmento (2003, p.3-4) como "a capacidade das crianças em construírem de forma sistematizada modos de significação do mundo e de açção intencional, que são distintos dos modos adultos de significação e acção". As culturas da infância são socialmente reproduzidas pelas crianças e são historicamente constituídas e reconstituídas, sendo este um processo em contínuo movimento. Também são constituídas na tensa relação entre gerações, ou seja, suas características formam-se entre as culturas geradas pelos adultos e as culturas construídas nas interações entre as crianças. É nessa tensão geracional que as crianças se encontram, disputando poderes e buscando desafiar e questionar a autoridade do mundo adulto, seja na escola, seja na família ou em outros espaços de convivência.

A infância é uma categoria estrutural presente em todas as sociedades (CORSARO, 2011). Mas, apesar disso configurar o termo "infância" como uma característica comum a todas as crianças, existe uma pluralidade nos modos de ser criança. E são esses diferentes modos de ser, que podem ser compreendidos a partir dos desenhos das crianças. Como pluralização das culturas infantis, Sarmento define:

A pluralização do conceito significa que as formas e conteúdos das culturas infantis são produzidas numa relação de interdependência com culturas societais atravessadas por relações de classe, de gênero e de proveniência étnica, que impedem definitivamente a fixação num sistema coerente único dos modos de significação e acção infantil. (SARMENTO, 2003, p.4)

Considerando a pluralidade dos modos de ser criança, podemos compreendêla a partir de alguns eixos estruturantes das culturas da infância, segundo Sarmento (2002, 2003). São eles: a interatividade, a ludicidade, a fantasia do real ou jogo simbólico e a reiteração.

A interatividade se exprime na sua forma mais intensa nas culturas de pares infantis (termo também utilizado por Corsaro (2011) e que será retomado mais adiante). O próprio processo de aprendizagem é interativo, na medida em que “[...] 
as crianças aprendem com as outras crianças, nos espaços de partilha comum" (SARMENTO, 2002, p.14). É interagindo entre pares - ou seja, entre crianças - que elas adquirem capacidade para se apropriar do mundo em que vivem, bem como reinventá-lo e reproduzi-lo. É nessa relação de convivência com outras crianças que se torna possível criar e reproduzir as características formadoras das culturas da infância de determinado grupo. Mas, não se podem deixar de lado as interações das crianças com o mundo dos adultos, pois é através dessas interações que se estabelecem as formas de controle dos adultos sobre as crianças, bem como formas de configurar o mundo das crianças - ambos (formas de controle e formas de configuração) sendo muito visíveis através de jogos e brinquedos.

Brinquedos, brincadeiras e jogos são momentos de interação entre as crianças, o que nos leva à característica lúdica das culturas infantis. É na natureza interativa do brincar que Sarmento (2002) diz constituir-se um dos primeiros elementos estruturantes das culturas da infância, pois, "o brinquedo e o brincar são também um factor fundamental na recriação do mundo e na produção das fantasias infantis." (SARMENTO, 2002, p.16).

Outros elementos fundamentais nas culturas infantis são a fantasia do real (ou jogo simbólico) e a reiteração. Sobre a fantasia do real, podemos considerá-la como uma forma de compreensão do mundo pelas crianças. É através da transposição imaginária de situações inventadas pelas crianças que elas elaboram formas de resistir ao mundo. A fantasia do real, então, permite à criança criar um mundo simbólico constituinte dela mesma enquanto sujeito.

E, por fim, a reiteração permite-nos compreender esse processo de imaginação do real, pois a criança possui a capacidade de se transportar no tempo, sem deixar de ser ela mesma, bem como a possibilidade de começar tudo de novo. O tempo da criança é um tempo não literal e não linear. É um tempo capaz de ser reiniciado e reinventado sempre que a criança sentir essa necessidade através do jogo simbólico e da fantasia do real.

Essa transposição do real através do jogo simbólico leva-nos às gramáticas das culturas da infância expostas por Sarmento (2002, 2003), enquanto traços distintivos que nos permitem compreender as culturas da infância e das crianças em 
várias dimensões. O termo "gramática" não limita o conceito a elementos linguísticos, mas serve como metáfora que permite identificar as regras de estruturação simbólica e os princípios de estruturação que dão sentido às características das culturas da infância.

\begin{abstract}
Assim, as culturas da infância podem ser analisadas na Semântica, isto é nos processos de referenciação e significação próprios das crianças, na Sintaxe, isto é nas regras de articulação entre os elementos simbólicos e na Morfologia, isto é, na especificidade das formas que assumem os elementos constitutivos das culturas da infância: os jogos, os brinquedos, os rituais, mas também os gestos e as palavras. Podem ainda ser analisadas na sua Pragmática, isto é, nas relações de comunicação que se estabelecem entre pares e nos modos pelos quais se realizam os processos de cooperação e de estratificação entre as crianças (ADLER e ADLER, 1999 apud SARMENTO, 2003, p.10)
\end{abstract}

Nosso esforço reside ainda, em buscar identificar os desenhos produzidos pelas crianças de acordo com as gramáticas estabelecidas por Sarmento. Nossa hipótese é de que o desenho expressa todas essas características fundamentais das gramáticas da infância. Pois os desenhos podem levar-nos a conhecer as referências e significados atribuídos pelas crianças a determinado tema (semântica). Podem mostrar-nos a articulação entre os elementos simbólicos desenhados pelas crianças em consonância com suas narrativas e suas projeções individuais em seus desenhos (sintaxe). Podem configurar as formas específicas de desenhar das crianças (morfologia) - obviamente diferente dos adultos, pois enquanto o adulto busca uma qualidade estética, a criança parece buscar uma qualidade narrativocomunicativa em seus desenhos. E por último, considerando a comunicação que é estabelecida entre seus pares na produção do desenho infantil, podemos observar a cooperação entre as crianças nos seus desenhos produzidos em grupos ou na observação do desenho do seu par e na importância que a opinião de outra criança possui (pragmática).

A capacidade que as crianças possuem de se recriar e se reinventar nas suas brincadeiras e desenhos, de se transpor em personagens sem deixar de serem elas mesmas, contribui para nossa compreensão dos desenhos infantis como uma expressão simbólica das crianças. As gramáticas das culturas da infância 
contribuem, assim, para nossa compreensão sobre as culturas da infância e os desenhos das crianças. Para aprofundar nossos conhecimentos, julgamos necessário continuar o diálogo, que faremos a seguir, através do conceito de reprodução interpretativa de Corsaro (2011).

\section{REPRODUÇÃO INTERPRETATIVA}

Conforme o exposto anteriormente, as culturas de pares infantis são importantes meios de interação das crianças. Pressupondo que através da interatividade entre seus pares as crianças sejam capazes de produzir e reproduzir cultura, conceituaremos a cultura de pares de acordo com Corsaro (2011). Este autor defende que as culturas de pares são um aspecto fundamental para compreender a reprodução interpretativa. Para Corsaro:

\footnotetext{
a cultura de pares é pública, coletiva e performática (GEERTZ, 1973; GOFFMAN, 1974). Portanto, em consonância com nossa abordagem interpretativa, defino cultura de pares infantis como um conjunto estável de atividades ou rotinas, artefatos, valores e preocupações que as crianças produzem e compartilham em interação com as demais (CORSARO; EDER, 1990 apud CORSARO, 2011, p.128)
}

Sendo assim, as culturas de pares possibilitam a produção e participação coletiva das crianças, tanto no mundo adulto quanto no mundo infantil. São nessas tensões entre o mundo adulto e o mundo infantil - tensões geralmente resultadas do poder dos adultos frente às crianças - que mesmo as crianças possuindo um papel ativo na produção cultural, ainda assim ocupam posições subordinadas em relação aos adultos. Isso vai ao encontro do que Corsaro (2011, p.129) considera ser o surgimento das culturas de pares: "[...] das tentativas infantis de dar sentido e, em certa medida, a resistir ao mundo adulto".

Tomando como ponto de partida esta compreensão da cultura de pares, torna-se possível compreender a noção de reprodução interpretativa. Esta se caracteriza como uma teoria desenvolvida por Corsaro em contraponto às teorias derivadas de concepções do desenvolvimento infantil que têm sido constantemente 
discutidas (por exemplo: visões tradicionais de socialização, teoria cognitiva, psicologia do desenvolvimento, noção de estágios do desenvolvimento etc.) para colaborar na construção de uma nova sociologia da infância. Eis, então, a noção de reprodução interpretativa:

o termo interpretativo abrange os aspectos inovadores e criativos da participação infantil na sociedade. Na verdade, [...] as crianças criam e participam de suas próprias e exclusivas culturas de pares quando selecionam ou se apropriam criativamente de informações do mundo adulto para lidar com suas próprias e exclusivas preocupações. O termo reprodução inclui a ideia de que as crianças não se limitam a internalizar a sociedade e a cultura, mas contribuem ativamente para a produção e mudança culturais. O termo também sugere que crianças estão, por sua própria participação na sociedade, restritas pela estrutura social existente e pela reprodução social. Ou seja, a criança e sua infância são afetadas pela sociedade e culturas que integram. Essas sociedades e culturas foram, por sua vez, moldadas e afetadas por processos de mudanças históricas. (CORSARO, 2011, p.31-32).

Com este termo, Corsaro busca destacar que as crianças, ao produzirem as culturas infantis, não estão apenas imitando o mundo adulto, mas sim se apropriando de forma criativa e produzindo e reproduzindo essas informações. Esse processo de apropriação criativa, denominado reprodução interpretativa por Corsaro, é composto por três tipos de ação coletiva. Primeiro: a apropriação criativa de informações e conhecimentos do mundo adulto pelas crianças. Segundo: a produção e participação de crianças na cultura de pares. E terceiro: a contribuição infantil para a reprodução e extensão da cultura adulta. De acordo com Corsaro (2002), podemos considerar, também, que o processo de reprodução interpretativa implica nas apropriações feitas pelas crianças, de forma criativa e ativa, dos modelos adultos que passam por um processo de embelezamento ou adaptação para depois serem confrontados com as realidades delas próprias.

Consideramos importante essa noção de reprodução interpretativa para o entendimento dos desenhos infantis, pois essa concepção nos permite compreender o processo de aquisição do conhecimento pelo qual as crianças passam para, então, produzirem seus desenhos. 
As crianças estão em determinado contexto e sob determinada pressão adulta, o que influenciará direta ou indiretamente no produto final do seu desenho. Conforme demonstrado por Corsaro (2011), vários pesquisadores utilizam os desenhos para provocar diálogos sobre histórias e entendimentos das crianças. Os desenhos, então, se configuram como uma forma de permitir a manifestação da criança sobre temas que normalmente ela possuiria dificuldade de expressar diante dos adultos, conferindo-lhe empoderamento nas pesquisas sociológicas.

Compreendendo as culturas de pares e as culturas infantis e, levando em conta o processo de reprodução interpretativa, podemos ter acesso às diferentes características das culturas infantis, sendo o desenho um método de acesso que permite às crianças expressarem suas próprias imagens e representações. Para isso, passamos às considerações teóricas sobre os desenhos infantis.

\title{
4 DESENHOS INFANTIS
}

Os desenhos infantis são, para Sarmento (2011), a produção simbólica de um grupo social de tipo geracional. Uma das importantes inovações da nova sociologia da infância é considerar que o desenho pode ser um importante canal e meio de comunicação não verbal. Conforme Sarmento:

\begin{abstract}
o desenho infantil insere-se entre as mais importantes formas de expressão simbólica das crianças. Desde logo, porque o desenho precede a comunicação escrita (na verdade, precede mesmo a comunicação oral, dado que os bebés rabiscam antes ainda de articularem as primeiras palavras). Depois, porque o desenho infantil, não sendo apenas a representação de uma realidade que lhe é exterior, transporta, no gesto que o inscreve, formas infantis de apreensão do mundo - no duplo sentido que esta expressão permite de 'incorporação' pela criança da realidade externa e de 'aprisionamento' do mundo pelo acto de inscrição - articuladas com as diferentes fases etárias e a diversidade cultural. Nesse sentido, o desenho infantil comunica, e fá-lo dado que as imagens são evocativas e referenciais de modo distinto e para além do que a linguagem verbal pode fazer. (SARMENTO, 2011, p.28-29).
\end{abstract}

Sarmento busca inserir o desenho infantil numa perspectiva socioantropológica, revelando não só a criança que desenha, mas também inserindo 
sua produção dentro do grupo geracional e do contexto no qual foi realizado. $O$ desafio colocado à sociologia, à antropologia e à educação é a "desconstrução da interpretação gradualista, descontextualizada e adultocêntrica" (SARMENTO, 2011, p.35) e a consideração do desenho infantil como uma produção simbólica, produto de diferenciados processos culturais de aprendizagens e condições de produção, que levam os desenhos a se transformarem em artefatos sociais e culturais, "testemunhos singulares de uma cultura que se exprime na materialidade dos produtos em que se comunica" (SARMENTO, 2011, p.36).

Sendo os desenhos infantis produtos simbólicos e artefatos sociais e culturais da infância, sua interpretação deve se dar na "polissemia das suas formas e cores" (SARMENTO, 2011, p.36-37). Em primeiro lugar, enquanto um produto singular do sujeito que o realizou - nesse caso a criança (devidamente contextualizada). Em segundo lugar, enquanto artefato social, repleto de significados sobre valores culturais (do contexto de determinada criança). E, por último (mas sem esgotar outras possibilidades), enquanto objeto simbólico que representa uma categoria específica - a infância - e suas subdivisões etárias, de acordo com suas capacidades gráficas diferenciadas.

Ainda, em relação à análise, devemos enfatizar a importância de levar em conta as condições sociais de produção, as condições sociais de existência das crianças e outras categorias sociológicas (por exemplo: classe, etnia, gênero, escolaridade etc.) relacionadas à categoria geracional "porque os diversos códigos culturais emanam dessas categorias" (SARMENTO, 2011, p.41).

O desenho infantil, considerado como uma forma de comunicação, em relação à cultura de pares e às culturas da infância, podem exprimir a interatividade e a comunicação entre as crianças no momento da sua produção em grupo. $O$ desenho também tem a característica de exprimir o mundo do faz-de-conta criado pelas crianças, evidenciando a importância de articular suas falas durante a produção do desenho, a fim de compreendê-lo na sua totalidade para evitar que se tirem conclusões errôneas: "o desenho infantil, afinal, é a expressão de uma das coisas que as crianças fazem de mais sério: brincar." (SARMENTO, 2011, p.51). 
Em outro trabalho de pesquisa - realizado para concluir o curso de Ciências Sociais (BORDIN, 2010), buscamos pesquisar os desenhos infantis produzidos durante as aulas de artes de uma turma de $5^{\text {a }}$ série do ensino fundamental de uma escola pública. $\mathrm{O}$ objetivo era entender como os alunos interagiam em sala de aula, como produziam e que significados atribuíam aos seus desenhos. A principal metodologia do trabalho consistia em uma análise semiótica (PEIRCE apud SANTAELLA, 2003), onde buscamos identificar signos nos desenhos produzidos, a fim de compreender a cultura dos alunos. "O signo é uma coisa que representa outra coisa, e ele possui alto poder representativo." (BORDIN, 2010, p. 38). Nessas representações, classificamos os signos enquanto índices, ícones ou símbolos a fim de melhor compreendê-los, buscando não classificá-los de forma a perderem seu significado original. Foi uma metodologia que nos possibilitou conhecer diferentes níveis de significados nos desenhos, desde ícones (signos sugestivos), passando por índices (signos relacionados a algo real e concreto) e símbolos (signos abstratos com alto poder representativo).

A escola se destacou como um contexto significativo para a produção dos desenhos das crianças (BORDIN, 2010). Em seus desenhos e interações verbais com os colegas, as crianças produziam e reproduziam as características das aulas, dos alunos, da escola e de si mesmos e suas famílias fora da escola. Representavam também temas e situações que durante o período da pesquisa se tornaram rotineiras, como por exemplo: a chegada à sala de aula, a troca de períodos, os problemas com outros alunos e com professores, a influência de programas televisivos, a cooperação entre os alunos no momento de produção dos desenhos, a partilha de opiniões etc. Essas situações surgidas durante a pesquisa de campo, formaram o sistema de relações que caracterizaram o ambiente escolar dos alunos. Puderam ser consideradas como culturas da infância em relação à escola, sendo que a forma como se manifestaram pode inseri-las nas características da reprodução interpretativa. Deste trabalho surgiu a hipótese de que as características formadoras da reprodução interpretativa são de grande utilidade para compreender o processo de criação dos desenhos infantis. 
Utilizamos este trabalho para exemplificar o quanto a escola pode condicionar a produção dos desenhos infantis e o quão importante é, para pesquisadores e professores, estarem atentos aos diálogos durante as atividades. Desenhos e diálogos juntos colaboram para revelar importantes aspectos culturais, livre de interpretações não fundamentadas, se transformando em importante ferramenta metodológica para quem trabalha (pesquisa ou docência) com crianças.

\begin{abstract}
Estes desenhos, enquanto traços realizados pelos sujeitos que os produziram são, antes de tudo, signos indiciários de seus autores, marcas deixadas no papel por contigüidade de seus gestos manuais, mas também são signos simbólicos, reveladores de um imaginário coletivo agindo sobre o imaginário individual e possibilitando identificar, por convenção, as referências que as imagens podem vir a representar. Sendo essas referências criadas a partir de estímulos externos de referentes que as crianças tentam imitar em sua aparência, elas também são signos icônicos, mas seus significados podem igualmente se transformar em símbolos generalizados e aplicados pedagogicamente pelos professores. Apesar da influência externa, esses referentes não se configuram como fatores exclusivamente objetivos para a produção dos alunos, pois cada criança, de sua maneira, identifica esses estímulos e os interpreta, traduzindo-os em signos repletos de significados subjetivos e intersubjetivos - condição mesmo da comunicação humana e manutenção de uma cultura. Deste modo a importância dos desenhos produzidos em sala de aula, veio possibilitar uma reflexão, não só sobre a sociedade mas também sobre a escola como tal. (BORDIN, 2010, p. 66)
\end{abstract}

A tese de Gomes (2009) teve como objetivo conhecer a cultura geracional da infância a partir do desenho infantil. Gomes considerou o desenho infantil enquanto uma produção simbólica e uma linguagem original das crianças, concretizando-se em veículo de comunicação. A autora buscou investigar a importância do contexto escolar na ação e produção dos desenhos das crianças, em que medida o desenho infantil seria um meio de comunicação inter e intrageracional, quais os indicadores de apropriação cultural presentes nas narrativas gráficas, as vivências significativas do autor do desenho inscritas na sua produção, e, a expressão da interpretação do real feita pela criança no seu desenho. As conclusões desse trabalho revelaram a produção dos desenhos infantis sob o condicionamento escolar, através do poder do adulto, assim como se configurou enquanto um meio de comunicação entre pares e com adultos. Essa produção foi também percebida enquanto uma linguagem simbólica expressiva dos mundos das crianças e portadora de elementos culturais. 
As crianças também inscreveram em seus desenhos as vivências de seus cotidianos, bem como o conhecimento que possuem sobre sua comunidade.

É importante destacar que o trabalho de Gomes (2009) foi uma pesquisa com base etnográfica e com utilização de registro escrito - diário de campo. Os desenhos foram produzidos na sala de aula ou sala de atividades em uma comunidade rural e periférica de Portugal, com crianças entre cinco e nove anos. A organização do trabalho entre as crianças se dava de duas maneiras: as crianças desenhavam sozinhas na sua mesa e conversavam com o colega ao lado e as crianças desenhavam numa mesa em grupo e trocavam ideias entre elas. As temáticas eram negociadas com a turma: podiam ser livres e sugeridas pelas crianças ou pela pesquisadora.

As conclusões do trabalho de Gomes nos fazem retomar as culturas da infância e a reprodução interpretativa, na medida em que, através dos seus desenhos, as crianças representaram suas culturas próprias. Quando seus desenhos são relacionados com as interações e diálogos (entre as crianças e com a pesquisadora), se torna possível perceber a apropriação criativa feita pelas crianças e reproduzida nas suas narrativas gráficas, indo ao encontro de algumas conclusões feitas na pesquisa anterior (BORDIN, 2010).

\section{CONTRIBUIÇÕES À PESQUISA E À PRÁTICA EDUCATIVA}

Apesar de serem poucas pesquisas sociológicas que têm como tema principal os desenhos das crianças, a partir das considerações expostas, e de nossos próprios trabalhos recentes de pesquisa (BORDIN, 2013; 2014), podemos concluir que estes têm sido uma forma de expressão simbólica das crianças reveladora de importantes conhecimentos sociais e que nos têm demonstrado a ação escolar e dos adultos sobre a produção do desenho infantil - muitas vezes sendo uma ação inibidora. Os desenhos têm-se mostrado também como importante fonte de comunicação (tanto entre as crianças, como entre crianças e adultos) e ainda, enquanto produções (de sujeitos específicos) que carregam consigo elementos culturais, sociais, econômicos e familiares presentes nas vidas das crianças, e, que 
estão ali - na sala de aula ou em qualquer outro contexto - prontos para serem decifrados e valorizados como importante fonte de conhecimento sobre a infância.

Para pesquisas científicas sobre as crianças em diferentes contextos, os desenhos podem ser uma importante ferramenta metodológica que possibilite revelar os aspectos culturais buscados pelos investigadores. E relacionados às teorias sociais escolhidas, os desenhos se efetivam como um importante campo de pesquisa que busca relacionar a Sociologia e a Educação Infantil, ainda em desenvolvimento, mas já com algumas indicações metodológicas importantes, conforme exposto por Sarmento (2011).

Para educadores preocupados em conhecer melhor seus alunos, os desenhos infantis também são importante fonte de conhecimento. Possibilitam aos professores expandir seu trabalho, agregando conhecimento cultural e social às suas aulas, partindo dos seus próprios alunos, bem como conhecer as condições à que seus alunos estão expostos, possibilitando inserir estas informações no planejamento das aulas.

As considerações sobre desenhos infantis também nos permitem pensar a Educação na medida em que representam aspectos educacionais possíveis e necessários de serem questionados. Para Sarmento (2011), os desenhos são uma produção simbólica das crianças, constituindo-se enquanto um meio de comunicação não verbal. O desenho possibilita à criança comunicar aquilo que por meio da fala ela não comunica. Permite também, aos adultos, entrar no âmago da infância e compreender (ou pelo menos tentar) a situação da educação nas escolas. Mesmo que os desenhos não tratem diretamente sobre esse tema, de certa forma eles comportam elementos que nos permitem pensar a educação; sendo assim, mais do que meros desenhos, são testemunhos de uma cultura infantil e escolar que parte do olhar das próprias crianças. O estímulo à criação de desenhos na educação infantil, seja individualmente ou em pequenos grupos, enfatiza o caráter interativo da infância bem como estimula também a ludicidade, a fantasia do real, o jogo simbólico e reiteração, aspectos trabalhados por Sarmento (2011).

O desenho torna possível a nós, adultos, compreender um outro mundo possível e desconhecido, revelado pelas crianças. Eles refletem um simbolismo ao 
mesmo tempo autônomo e condicionado socialmente. O desenho se configura então, não como uma tradução do mundo da criança, mas como uma inscrição de um ato e de um sujeito específico.

Com isso, os novos estudos sociais da infância, focados nas culturas infantis e na reprodução interpretativa, independente da metodologia, se mostram de extrema importância ao trabalho docente, pois conforme Sarmento (2011) e Corsaro (2011), permitem aos adultos compreender o significado partilhado entre as crianças sobre o mundo que as envolve.

\section{CONSIDERAÇÕES FINAIS}

Os desenhos infantis têm se revelado importante meio de acesso às culturas infantis e suas análises um importante meio de acesso à maneira como as crianças se apropriam das culturas adultas e produzem suas próprias culturas (reprodução interpretativa). Ter conhecimento sobre as características que formam a cultura de determinado grupo, propicia aos envolvidos neste grupo - professores ou pesquisadores - maiores conhecimentos e possibilidades metodológicas de trabalho. Ainda é um desafio para os estudiosos dessa área de pesquisa elaborar metodologias de acordo com as necessidades das crianças. Precisamos de metodologias que captem a integralidade das crianças na produção dos seus desenhos. E é apelando à nossa imaginação, que podemos criar esses métodos que muitas vezes surgem no contato direto com nossos sujeitos de pesquisa, durante o trabalho de campo.

A sociologia de Corsaro $(2002 ; 2011)$ e de Sarmento $(2002 ; 2003 ; 2011)$ nos apontam os aspectos interativos das crianças na formação de suas culturas. Essas interações são constituídas por ações coletivas que criam e reforçam os laços sociais entre as crianças, possibilitando a elas criarem formas de resistência ao mundo social que as envolve, em especial atenção à escola e aos métodos tradicionais de ensino (copiar, decorar).

O desenho é resultado de um desenvolvimento motor específico de cada criança e consideramos que não cabe a nós, sociólogos, analisá-los como se 
fossem tipos ideais. Tal perspectiva seria para nós um equívoco, pois, cairíamos naquilo que estivemos tentando evitar: a classificação e estereotipação do desenho infantil. Mais do que uma representação das fases do desenvolvimento humano, o desenho infantil empodera as crianças e facilita a comunicação entre crianças e adultos. Mas, para haver essa comunicação, é necessário largar nossas préconcepções e mergulhar nas aventuras que as crianças contam enquanto desenham. É nesse momento que o simbolismo floresce e as crianças desenvolvem seu raciocínio lógico, sua imaginação criadora e seus conhecimentos sobre o mundo.

Finalizamos considerando que os desenhos infantis também podem ser uma forma de crítica à cultura, na medida em que expressam a opinião das próprias crianças sobre determinado assunto e permite-nos ampliar nosso conhecimento sobre as peculiaridades da infância contemporânea nas diversas categorias sociológicas possíveis de pesquisar. Consideramos os desenhos, então, como fonte metodológica fundamental para pesquisar sobre a infância, pois os desenhos revelam além do sujeito que o produziu, também os valores culturais que o sujeito carrega, bem como as condições sociais de produção e existência das crianças. Isso porque, durante a produção de seus desenhos, a criança se manifesta livremente, fazendo de sua criação algo propulsor para outros debates e diálogos entre seus pares e com os adultos.

\section{FRANCINE BORGES BORDIN}

Mestre em Educação pela Universidade Federal de Pelotas (UFPel).

\section{DENISE BUSSOLETTI}

Doutora em Psicologia pela Pontifícia Universidade Católica do Rio Grande do Sul (PUC-RS). Professora Associada da Universidade Federal de Pelotas (UFPel) e atual Pró-reitora de Extensão e Cultura dessa mesma universidade.

\section{REFERÊNCIAS}


BORDIN, F. B. As representações expressas nos desenhos infantis: um olhar antropológico. 2010. 75 p. Monografia (Bacharelado em Ciências Sociais) Universidade Federal de Pelotas, Rio Grande do Sul.

. A sociologia da infância e os desenhos infantis: uma contribuição sociológica à educação. 2013. 18 p. Trabalho de Conclusão de Curso (Especialização em Educação infantil) - Universidade Federal de Pelotas, Rio Grande do Sul.

. "Não é de verdade, é só um desenho": de que nos falam os desenhos infantis? 2014. 171 p. Dissertação (Mestrado) - Programa de Pós-Graduação em Educação, Faculdade de Educação, Universidade Federal de Pelotas, Rio Grande do Sul.

CORSARO, W. A. A. reprodução interpretativa no brincar ao "faz-de-conta" das crianças. Educação, Sociedade e Culturas, Porto, n.17, p.113-134, 2002. Disponível em: <http://www.fpce.up.pt/ciie/revistaesc/> Acesso em: 19 set. 2009.

. Sociologia da Infância. Porto Alegre: Artmed, 2011, 384 p.

REVISTA EDUCAÇÃO E SOCIEDADE. Dossiê "Sociologia da Infância: pesquisas com crianças. v.26, n.91, maio/ago.2005. Disponível em:

$<$ http://www.scielo.br/scielo.php?script=sci_issuetoc\&pid=0101-

733020050002\&lng=pt\&nrm=iso > Acesso em: março de 2010.

GOMES, Z. F. F. Desenho infantil: Modos de interpretação do mundo e simbolização do real. Um estudo em Sociologia da Infância. 2009. 187 p. Dissertação (Mestrado em Sociologia da Infância) - Universidade do Minho, Portugal. Disponível em: <http://hdl.handle.net/1822/11016>. Acesso em: 10 mar. 2011.

SANTAELLA, L. O que é semiótica. 2. ed. São Paulo: Brasiliense, 2003, 86 p.

SARMENTO, M. J. As culturas da infância nas encruzilhadas da $2^{a}$ modernidade. Instituto de Estudos da Criança. Universidade do Minho. 2002. Disponível em: $<$ http://cedic.iec.uminho.pt/Textos_de_Trabalho/menu_base_text_trab>. Acesso em: 22 set. 2009.

. Imaginário e culturas da infância. Instituto de Estudos da Criança.

Universidade do Minho. 2003. Disponível em:

<http://cedic.iec.uminho.pt/Textos_de_Trabalho/menu_base_text_trab.htm>. Acesso em: 15 set. 2009.

. Conhecer a infância: os desenhos das crianças como produções

simbólicas. In: MARTINS FILHO, A. J.; PRADO, P. D. Das pesquisas com crianças à complexidade da infância. Campinas, São Paulo: Autores Associados, 2011. p. 2760 . 
Atos de Pesquisa em Educação - ISSN 1809-0354

Blumenau, v. 9, n.3, p.681-698, set./dez. 2014

DOI: http://dx.doi.org/10.7867/1809-0354.2014v9n3p681-698

SARMENTO, M.; GOUVEA, M. C. S. (Orgs). Estudos da Infância: educação e práticas sociais. Petrópolis: Vozes, 2008, 384 p. 